\title{
Investigating the translational and rotational motion of the swing using accelerometers for athlete skill assessment
}

\author{
Amin Ahmadi, David D. Rowlands, Daniel A. James \\ Centre for Wireless Monitoring Applications \\ Griffith University, School of Engineering \\ Brisbane, Australia \\ d.james@griffith.edu.au \\ amin.ahmadi@student.gu.edu.au
}

\author{
Amin Ahmadi \\ Centre of Excellence \\ Queensland Academy of Sport \\ Brisbane, Australia
}

\begin{abstract}
In this paper, an accelerometer measurement system comprising three accelerometer nodes was used to identify the correlation between the skill level and the characteristics of the first serve swing in tennis. Three MEMS accelerometers were mounted on the knee, leg, and wrist of the tennis players. The kinematic model for the first serve was observed. Furthermore, this study revealed that side-forward motion of the hand along with the forward motion of the waist of an athlete can be used as indicator to assess the athlete's skill level. It is envisaged that this application can provide feedback to tennis players.
\end{abstract}

\section{INTRODUCTION}

The serve is the most important $[1,2]$ and critical stroke in tennis. It is well known that fast serves can dominant the game at elite levels [1]. However, it is the most complex stroke as there are almost nine contributors between maximum knee flexion and impact involved [3] to produce the fast serve. As a result, powerful serves can not be performed without the correct biomechanical movements of different segments of the body. Some studies have been done on the kinematic model $[4,5,6]$ as well as the kinetic model of the serve $[7,8,9]$. All of them are based on videography and optical monitoring techniques but do not use accelerometers.

Acceleration sensor technology has been used to analyse similar kinetic processes for golfer's lateral swing [10] and Aikido sword cut [11]. Accelerometer technology is getting more popular as it has some advantages over the previous method. Using accelerometers eliminates the need that experiments be carried out solely inside the laboratory to obtain real-time feedback which means that experiments can be run in the real environment. Accelerometers are small and light enough to be placed on any part or segment of the body without hindering performance. They are also very cheap compared to the other technologies. Therefore, swing analysis using accelerometers is becoming an area of increasing interest and importance. Within many sports applications, accelerometers are now used to measure and classify activity and effort levels. For instance, the key characteristics of the basic swing of Japanese swordsmanship can be extracted using accelerometers [11]. Accelerometers have also been proven to be capable of detecting the kinematic chain in golf lateral swing [10]. In that study, golfers with different levels of proficiency were categorized once the swing was performed. Using the same technology, it is possible to study the tennis serve motion.

The purpose of this study is to use accelerometers to examine the tennis player's serve during the backswing preparation as well as the forward swing motions. Kinematic chain pattern and correct angular and translational movements of the knee, waist and wrist of the player which are critical to maximize the ball velocity at impact will be discussed.

\section{Methodology}

The accelerometer measurement system which has been previously described in [12] is improved in a way that it is equipped with $\pm 10 \mathrm{~g}$ accelerometer nodes to capture faster movements. The three channels are also better synchronized than the previous system described in [12].This system includes a microprocessor board, Bluetooth connection, onboard and off-board ADXL202 and ADXL210 accelerometer sensors, and a VB receiving client running externally. The specification of the system is summarized in table 1 .

Three distinct accelerometer nodes were employed to capture the kinematic chain during the serve. The position and coordination of sensors when a player is facing the court is shown in figure 1. As it is demonstrated in figure 1, the accelerometer nodes are attached on the knee, waist, and wrist of the tennis player. Low-g MEMS accelerometers (ADXL202, $\pm 2 g$, Analog Devices) were applied on the 
knee and the waist and a middle MEMS accelerometer (ADXL210, $\pm 10 \mathrm{~g}$, Analog Devices) was attached on the wrist due to higher acceleration of the racquet-arm movement than the other nodes.

Four male tennis players with different levels of skill participated in this research. Two of which were sub-elite players and the rest were recreational-developmental players. Each player was allowed to take as much time as needed to warm up. Once the player was ready, the accelerometer system was mounted on three different spots (knee, waist, and wrist) of his body and then he was asked to perform approximately 10 first serves to ensure that he felt comfortable with the system and the system was not limiting his movements. Each player was then asked to serve from the deuce side and come back to his original position after each serve. He was then asked to delay for approximately 5 seconds before starting the next serve. This allowed us to recognize the serve's signals from other movement's signals and helped the player to focus on his next serve. Two digital video cameras (30fps) were also recording film to capture the movement. The accelerometer nodes were synchronized with the recorded images to determine the kinematic action. So that specific events could be identified and then a model developed from the acceleration data.

TABLE I. HARDWARE SPECIFICATION OF THE SENSOR MONITORING SYSTEM

\begin{tabular}{|l|l|}
\hline \multicolumn{1}{|c|}{ Feature } & \multicolumn{1}{c|}{ Value } \\
\hline Resolution (Accelerometers) & 10 bits \\
\hline Sampling Rate $@$ 3 channels & Up to $500 \mathrm{~Hz}$ per channel \\
\hline Accelerometer Range & $\pm 2 \mathrm{~g}, \pm 10 \mathrm{~g}$ \\
\hline Data Transmission Type & Wireless (Bluetooth 1.1) \\
\hline Transmission Range & Up to 250 metre \\
\hline
\end{tabular}

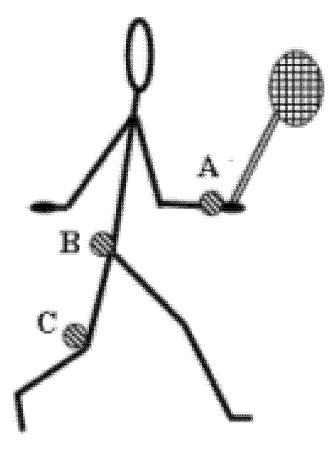

(a)

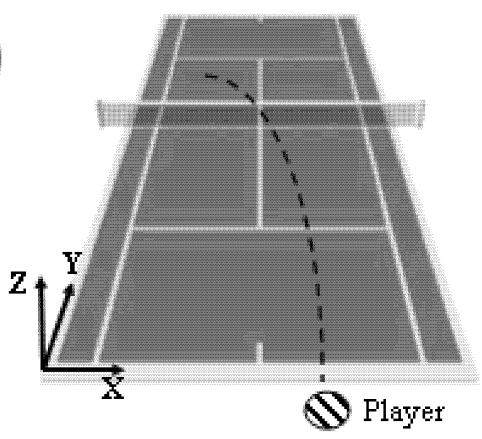

(b)
Figure 1. (a):Location of sensors on the athlete's body, (b): Acceleration coordinates when facing the tennis court

\section{RESULTS AND DISCUSSION}

Average acceleration values for each of the sensors for each athlete were used to determine the trends so that the effects of variations between individual serves were minimised. The average value was determined from the 10 serves performed by the athlete as part of the experimental protocol. The standard deviation was also used as an indicator of repeatability of the service action. The impact time was determined from the video and the sensor data.

(a) Hand
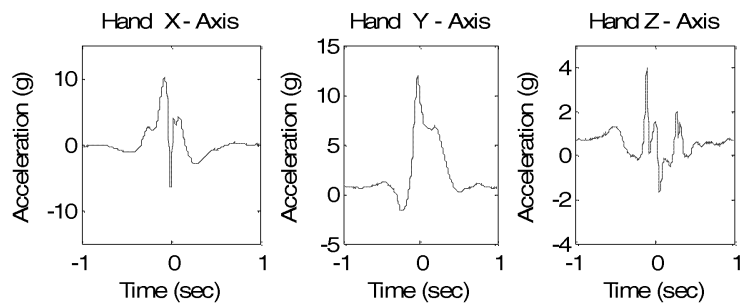

(b) Waist
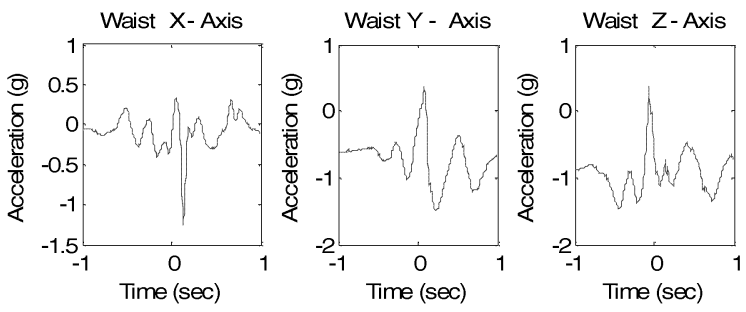

(c) Leg
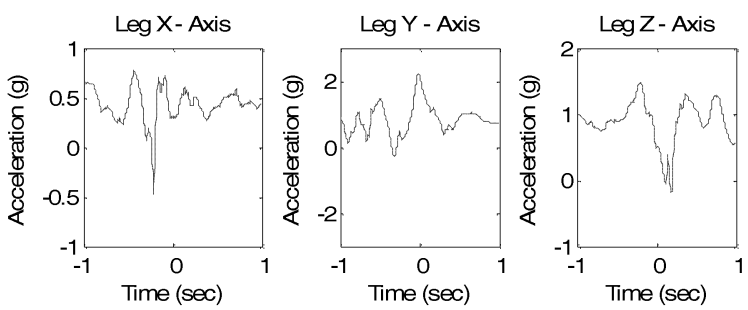

Figure 2. The average of 10 serves performed by a sub-elite player. (a) presents hand motions in $\mathrm{X}, \mathrm{Y}, \mathrm{Z}$ axis, (b) presents waist motion in $\mathrm{X}, \mathrm{Y}, \mathrm{Z}$ axis, and (c) presents leg motion in $\mathrm{X}, \mathrm{Y}, \mathrm{Z}$ axis

The average acceleration values for the leg, waist and wrist of the sub-elite tennis player are shown in figure 2 . In this figure time zero represents the impact time.

Both side and forward motion of the hand prior and after impact time are shown clearly. The $\mathrm{X}$ and $\mathrm{Y}$ axis show a relatively high peak. This is partly due to the rotation of the lower and upper trunk as well as the shoulder rotation needed to produce the circular motion of the racquet during the serve $[1,2,3]$. The $y$-axis graph is also due to the weight shift of the body in the forward motion of the serve.

It can also be seen in figure 2 that the peak of the forward waist acceleration occurs around the impact time as the body is fully rotated toward the court to hit the ball. This is most 
apparent in both the $\mathrm{X}$ and $\mathrm{Y}$ axis due to the waist rotating in that plane.

The knee flexion and stretch are also apparent in the acceleration of the leg.

The $\mathrm{Z}$ axis of the wrist, waist and leg all indicate the upward motion of the entire body in the serve. The $Z$ axis of the waist and the hand show the upward motion of the trunk as the athlete reaches the full height for the serve.

\section{A. The Kinematic Chain}

According to the kinematic chain model of serving to produce the fast (first) serve, the sequential order of the joint contributors start from the ground and goes all the way up to the wrist $[1,3]$. This sequence can be seen in figure 2 from the timing of the events prior to impact. The $\mathrm{X}$ and $\mathrm{Y}$ axis for the leg show that the knee flexion and knee full stretch occur around 0.25 seconds and 0.012 seconds prior to the impact respectively. The rotation of the waist occurs 0.08 seconds before the impact. Finally, the impact occurs at time zero.

The order of motion starts at the leg, proceeds through the waist until the impact. This follows the expected kinematic chain. Therefore this indicates that accelerometers can be successfully used to detect the body motion.

\section{B. Investigating the level of Proficiency of different players}

As shown in the previous section accelerometers can be used to detect the action of the swing for a tennis player. Therefore measurement of the swing action will makes it possible to compare the serves of athletes with different levels of skill. It is envisaged that this can be used to give for feedback to a tennis player and coach.

Figures 3 and 4 show selected sensor measurements for players with different skill levels. The players are of the subelite level denoted P1 and P2 and the amateur-developmental level denoted A1 and A2. The selected measurements are the side motion of the hand as shown in figure 3 and the forward motion of the waist as shown in figure 4. As can be seen, players with different level of skill exhibit different swing patterns.

The most indicative motion of the hand can be seen in the side motions. It can be seen that there are differences in the pattern of the side motion for sub-elite and amateurs. The differences are most apparent around the impact. Since the amateur player doesn't employ his waist to generate power to the serve, his side motion pattern is completely different from that of the sub-elite player. There is also a significant difference in acceleration magnitude between the amateurs and sub-elite players around impact time.

The forward waist motion also exhibits a difference between the sub-elite and the amateur. This is shown in figure 4 . The forward motion of the waist is linked to the rotation of the waist in the service motion. The rotation pattern is varying from the sub-elite players to the developmental players. It shows that for the developmental players there is either no rotation or some rotation at the wrong time. In contrast, the full rotation occurs around the impact time for the sub-elite players.

Repeatability is another key feature that may be used to classify the athletes. The grey lines in figures 3 and 4 show the standard deviation for each player. As can be seen, the sub-elite players have less variability through this whole swing than the developmental players. This indicates that the sub-elite players are more consistent in their service and their action is more controlled. It should also be noted that the standard deviation alone can not be used to detect the correct motion as a player might consistently repeat a bad habit. Therefore, using both the standard deviation and the average of the swing pattern can be used for an indication of the proficiency level of an athlete.
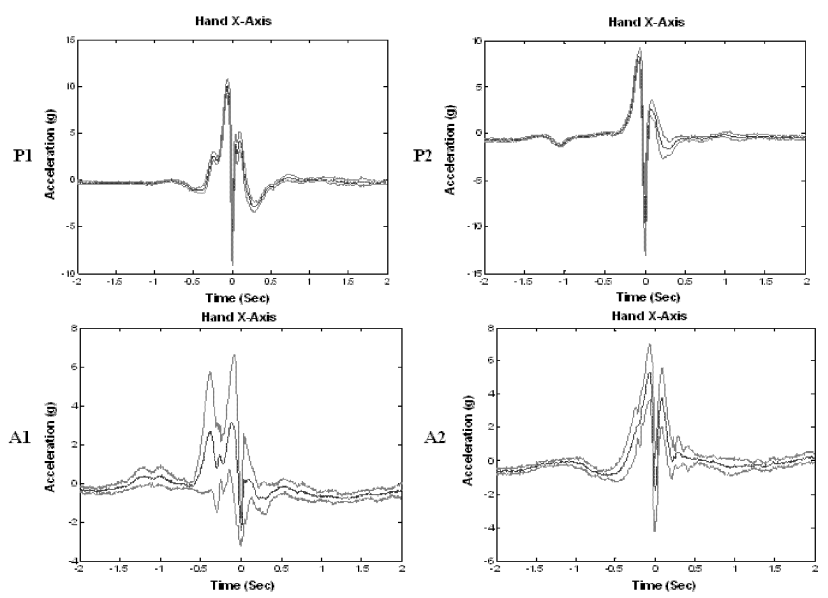

Figure 3. Average (dark line) and average \pm standard deviation (grey line) of the hand side motion for 2 sub-elite and two developmental players
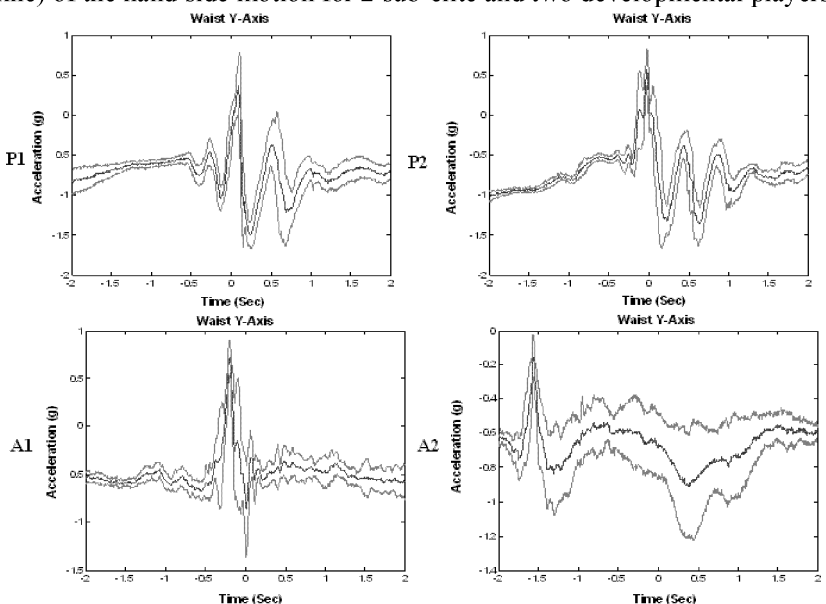

Figure 4. Average (dark line) and average \pm standard deviation (grey line) of the waist forward motion for 2 sub-elite and two developmental players. 


\section{CONCLUSION}

The focus of the paper was to use accelerometers to determine the motion during a tennis serve and then determine indicators of skill level for athlete skill assessment. The authors modified the previous [12] accelerometer measurement system to be able to record the higher acceleration of the hand as well as to obtain better synchronization of the measurements. The correct sequential order of the kinematic model of the tennis serve was observed using the accelerometer sensors. In addttion, differences between sub-elite athlete's serves and developmental athlete's serves were investigated. The most obvious differences between the sub-elite athlete's serves and developmental athlete's serves were detected in the hand side-forward motions and the waist forward motions. The waist forward motion is an indicator of the waist rotation. The repeatability of the swing was also examined. As expected, the developmental athletes have larger variability through the whole swing than the sub-elite athletes.

Overall this paper has shown that accelerometers can be used to monitor the kinematic model as well as give indicators towards assessing athlete's skill level.

\section{ACKNOWLEDGMENT}

The authors would like to thank the Queensland Academy of Sport (QAS) and the Centre of Excellence (COE) for funding this work.

\section{REFERENCES}

[1] Biomechanical Principals of Tennis Technique Using Science to Improve Your Strokes, chapter three

[2] E. Bahamonde, Changes in angular momentum during the tennis serves, Journal of Sports Sciences, 579-592, 2000
[3] J. Brian, D. Jesus, Contribution of joint rotations to racquet speed in the tennis serve, Journal of Sports sciences, 31-49, 2006

[4] R. Sprigings, B. Marshall, B. Elliott and L. Jennings, A threedimensional kinematic method for determining the effectiveness of arm segment rotations in producing racquet-head speed. Journal of Biomechanics, 27, 245- 254, 1994

[5] B. Van Gheluwe, M. Hebbelinck, . Kinematics of the service movement in tennis: A three-dimensional cinematographical approach. In Biomechanics $I X-B$ (edited by D. Winter, R. Norman, R. Wells, K. Hayes and A. Patla), pp. 521- 525, 1985

[6] B. Van Gheluwe, M. Hebbelinck, Muscle actions and ground reaction forces in tennis. International Journal of Sport Biomechanics, 2, 88-99, 1986

[7] R.E. Bahamonde, . Biomechanical analysis of serving during the performance of flat and slice tennis serves. Unpublished doctora dissertation, Indiana University, 1994

[8] G. Legnani, R.N. Marshall, Evaluation of the joint torques during the tennis serve: Analysis of experimental data and simulations. In Proceedings of the VIth International Symposium on Computer Simulation in Biomechanics, pp. 8- 11. Paris: International Society of Biomechanics, Technical Group on Computer Simulation. , pp 811,1993

[9] B. Elliott, G. Wood, The biomechanics of the foot-up and foot-back tennis serves techniques. Australian Journal of Sports Sciences, 3, $3 \pm$ 5,1983

[10] Y. Ohgi, T.Baba, Uncock Timing in Driver Swing Motion, The Impact of Technology on Sport,. AST A, 349-354, 2005

[11] James, D., Gibson, T. and Uroda, W,Dynamics of a swing: A study of classical Japanese swordsmanship using accelerometers, The Impact of Technology on Sport, Subic. A., Ujihashi, S. ASTA, 355-60, 2005

[12] James, D., Jaffari, I., A system for Multi-Limb Motion Monitoring Using Tri-Axial Accelerometer Nodes on a Wireless Network, The Impact of Technology on Sport,. ASTA, 361-66, 2005 